\title{
Omphalitis with Umbilical Abscess in an Adult with a Urachal Remnant
}

\author{
Antonios Tawk ${ }^{a} \quad$ Ali Abdallah ${ }^{b} \quad$ Paul Meouchy ${ }^{a} \quad$ Joanna Salameh ${ }^{c}$ \\ Salem Khoury ${ }^{d}$ Melissa Kyriakos $^{\mathrm{e}}$ Ghadi Abboud $^{\mathrm{b}}$ Mona Dagher $^{\mathrm{a}}$ \\ Peter Semaan $^{a}$ Aida Metria $^{\mathrm{a}}$ Raja Ashou ${ }^{\mathrm{b}}$ \\ ${ }^{a}$ Faculty of Medicine and Medical Sciences, University of Balamand, St. George Hospital \\ University Medical Center, Beirut, Lebanon; bepartment of Radiology, University of \\ Balamand, St. George Hospital University Medical Center, Beirut, Lebanon; 'Department of \\ Pathology, University of Balamand, St. George Hospital University Medical Center, Beirut, \\ Lebanon; ${ }^{d}$ Department of Gastroenterology and Hepatology, University of Balamand, \\ St. George Hospital University Medical Center, Beirut, Lebanon; 'Department of General \\ Surgery, University of Balamand, St. George Hospital University Medical Center, Beirut, \\ Lebanon
}

\section{Keywords}

Omphalitis · Umbilical abscess · Urachal remnant

\section{Abstract}

Omphalitis is an inflammation of the umbilicus and is seldom diagnosed in adults. It is even rarer when it results from an infection of the urachus, an embryological remnant that connects the umbilicus to the dome of the bladder. Patients with omphalitis present with erythema, edema, tenderness, and purulent discharge form the umbilical stump. Workup includes ultrasonography and CT scan of the abdomen and pelvis. Management consists of antibiotics and incision and drainage of the umbilical abscess, followed by surgical resection of the urachal remnant. In this article, we report a case of omphalitis complicated by umbilical abscess in a 20 -year-old female with a urachal remnant. 


\section{Introduction}

Omphalitis is the infection or inflammation of the umbilicus and its adjacent structures, and it typically affects the neonates [1,2]. Omphalitis among the adult population is described in the literature only as few case reports. It can result from an infectious process of a urachal remnant, the vitelline duct, or a foreign body in the umbilicus such as a navel piercing [3]. Embryologically, the urinary bladder wall is connected to the umbilicus through the allantois [4]. During fetal development, the allantois involutes and a fibrous remnant remains positioned in the preperitoneal space of Retzius [4]. This fibrous cord is called the urachus (or the median umbilical ligament of the adult), and it joins the dome of the bladder to the umbilicus [4]. Incomplete involution of the allantois can precipitate several urachal malformations, depending on the location of the disruption which may present as patent urachus, urachal cyst, umbilical-urachal sinus, vesico-urachal diverticulum, or alternating sinus [5]. The presence of such urachal remnants in an adult can predispose the patient to omphalitis.

The hallmark of omphalitis is a purulent discharge from the umbilical stump with surrounding edema, erythema, induration, and tenderness to palpation [2]. It is typically a polymicrobial infection that predominantly involves Staphylococcus aureus, Streptococcus pyogenes, Escherichia coli, Klebsiella pneumoniae, and Proteus mirabilis [2]. In patients with suspected omphalitis, a laboratory workup consisting of complete blood count and culture of the purulent material should be ordered. Ideally, the culture is obtained before the initiation of the antibiotic regimen [2].

The radiologic workup of omphalitis includes ultrasound of the umbilical area and computed tomography (CT) scan of the abdomen and pelvis [3]. Ultrasonography may show thickening of the fascia and fluid accumulation between subcutaneous fat and muscle [6]. CT of the abdomen and pelvis may determine the presence and extent of muscle and fascial involvement [6]. These imaging modalities are essential for the detection of any congenital anatomic abnormalities and to narrow down the differential diagnosis of omphalitis, which includes folliculitis, carbuncle, and pilonidal cyst in the umbilical area.

Most patients with omphalitis and a urachal remnant undergo a 2-staged approach in therapy [7]. The initial management is medical and consists of an antibiotic regimen that targets both Gram-positive and Gram-negative bacteria [7]. The choice of antibiotic can then be adjusted according to culture results. If an abscess is found on the physical exam and imaging, it should be incised and drained. The second stage of treatment is surgical resection of the urachal remnant because its presence can predispose to recurrent omphalitis [3]. In the following, we present a case of omphalitis complicated by an umbilical abscess in an adult with a urachal remnant.

\section{Case Presentation}

A 20-year-old female patient with no prior pertinent medical history presented to the Emergency Department at St. George Hospital University Medical Centre (Beirut, Lebanon) for a 2-week history of periumbilical pain. The patient described her pain throughout the 2 weeks as constant and nonradiating. Apart from anorexia, the patient had no fever, nausea, vomiting, diarrhea, or lower urinary tract symptoms. Three days prior to her presentation, the patient's periumbilical pain further increased in intensity and was, at that time, associated with severe chills; yet, no documented fever to be reported. Upon physical examination, the patient appeared to be in acute moderate distress. Her vital signs showed hemodynamic stability but a body temperature of $39.3^{\circ} \mathrm{C}$. Abdominal examination showed erythema and edema in the periumbilical area accompanied by severe tenderness to 

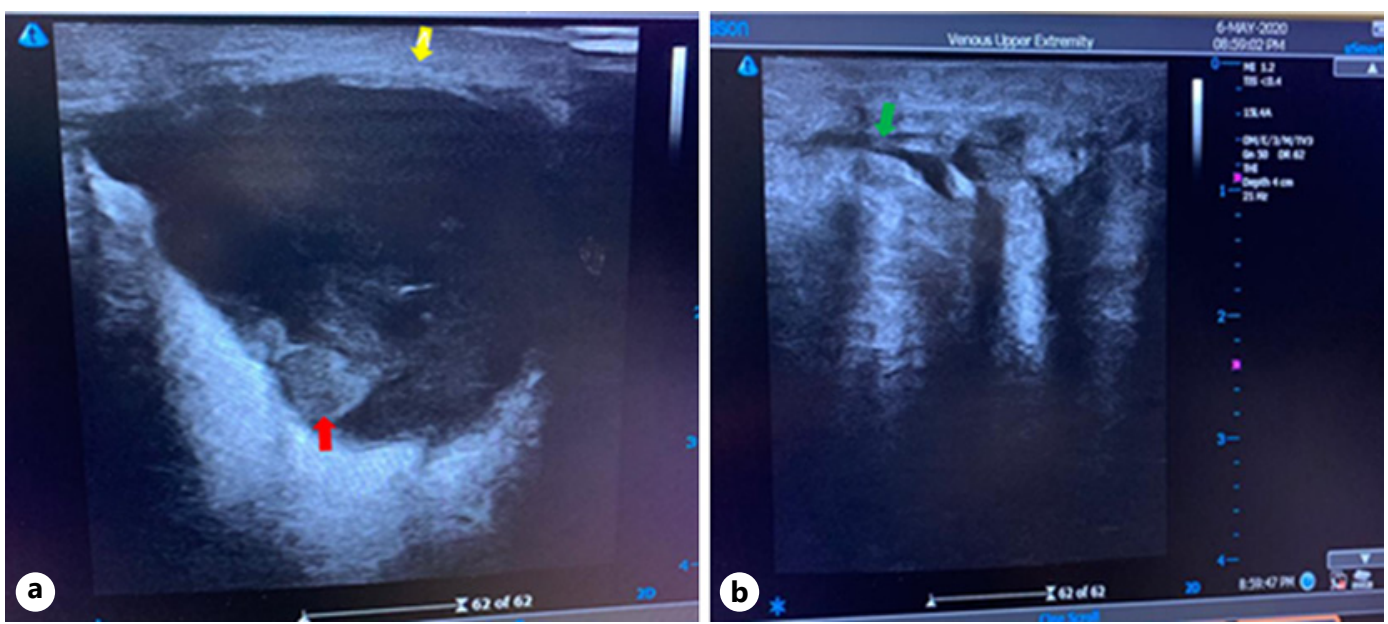

Fig. 1. Axial (a) and sagittal (b) enhanced images at the level of the umbilicus show a focal inflammation and localized abscess collection at the umbilicus and a tract in the abdominal wall measuring $3.3 \times 2.4 \mathrm{~cm}$, extending along $5 \mathrm{~cm}$ (yellow arrows). It is associated with infiltration of the surrounding fat plane, thickened skin, and a narrow umbilical neck (best seen on sagittal image, red arrow).

palpation and focal rigidity. However, no discharge was noted. Furthermore, the patient did not show any signs of peritoneal irritation. A digital rectal exam was performed and was negative for any tenderness or blood. A complete blood count with differential revealed leukocytosis of 13,400/L white blood cells counts, with a neutrophilic predominance of $85 \%$. Laboratory evaluation was negative for the biochemical studies and urine analysis. Moreover, the urine culture was negative for bacterial growth. The upright abdominal $\mathrm{X}$-ray was normal.

An ultrasound of the umbilical area showed skin thickening and a well-defined heterogenous area measuring around $3.3 \mathrm{~cm}$, extending from the umbilicus deep to the rectus musculature in the midline. An echogenic focus was also isolated posteriorly representing air (shown in Fig. 1a). Moreover, the longitudinal scanning over the umbilicus demonstrated a hypoechoic tract (shown in Fig. 1b). Further evaluation by a CT scan of the abdomen was performed, showing focal inflammation with localized abscess collections of the umbilicus measuring 3.3 $\times 2.4 \mathrm{~cm}$ extending along $5 \mathrm{~cm}$ in length. There was associated infiltration of the surrounding fat plane, thickened skin, and a narrow umbilical neck with a urachal remnant on the sagittal view. The final diagnosis was that the inflammation was the result of omphalitis with abscess formation or umbilical abscess (shown in Fig. 2). As such, empiric treatment with parenteral broad-spectrum antibiotics for the coverage of both Gram-positive and Gram-negative organisms was initiated. The patient was administered a combination of penicillin and an aminoglycoside for a total period of 10 days. Antibiotic treatment was accompanied with incision and drainage of the umbilical abscess.

\section{Discussion}

Urachal abnormalities are very rare. The incidence of urachal remnants in the pediatric population is 2 cases per 300,000 admissions to the pediatric hospital [8], and it is even lower in the adult population. These abnormalities are first diagnosed between the ages of 20 and 40 years, with a male-to-female ratio of 2:1 [8]. They are usually asymptomatic and only found incidentally on unrelated imaging or autopsy [8]. 

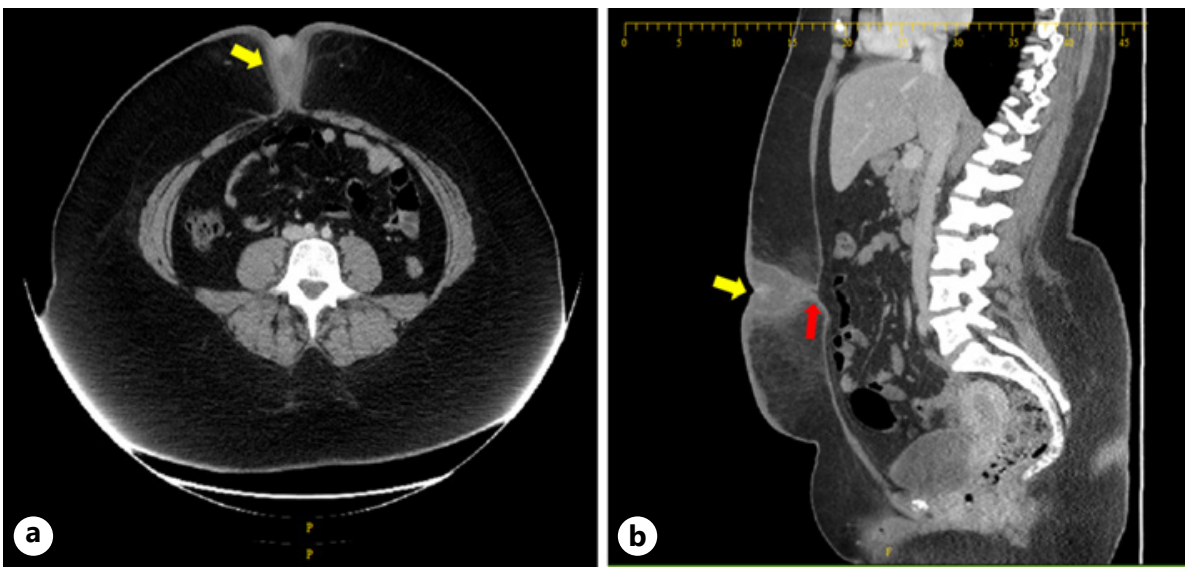

Fig. 2. a Transverse scanning over the umbilicus demonstrates a well-defined heterogenous area showing echogenic foci posteriorly representing air (red arrow). Re-demonstration of skin thickening (yellow arrow). b Longitudinal scanning over the umbilicus demonstrates a hypoechoic tract (green arrow).

The anatomy of the urachus and its relationship with the surrounding structures is important to consider in determining the signs and symptoms of omphalitis. The urachus and the bilateral umbilical arteries are located in the space of Retzius, a pyramidal fascial space that extends from the bladder dome to the umbilicus [4]. Because this space is preperitoneal, any infection involving the umbilicus or a urachal remnant will remain confined as a midline infraumbilical abscess, with no signs of peritonitis [8].

Erythema, edema, and tenderness to palpation of the umbilicus should raise suspicion of infection at the level of the umbilical stump [1]. Discharge from the umbilicus may or may not be present. If these signs of omphalitis are associated with fever, lethargy, and decreased oral intake, systemic involvement should be suspected [1]. The presented patient had a typical presentation of omphalitis, butno discharge was noted. She also had mild systemicinvolvement with fever and decreased oral intake.

The workup of omphalitis in adults includes ultrasonography and CT scan of the abdomen and pelvis. These imaging modalities demonstrate urachal lesions that are associated with omphalitis. In fact, an ultrasound alone is sufficient to diagnose a urachal remnant [9]. This remnant is described as an elliptical, hypoechoic structure that is located posterior to the rectus abdominis and anterosuperior to the bladder [10]. If an abscess is seen within the extraperitoneal fat space of the abdominal wall and extending to the umbilicus, it is a clue for the diagnosis of urachal abscess [11]. Therefore, the ultrasound has a high diagnostic accuracy and is considered the initial recommended test in diagnosing both omphalitis and urachal remnants [3]. If ultrasound results are inconclusive and second imaging modality is needed, a CT scan may be used. The CT scan is also beneficial when a malignancy of the urachal remnant is suspected [9]. Our patient underwent ultrasound and CT scanning of the abdomen and pelvis. Both imaging results showed omphalitis with abscess formation and the presence of a urachal remnant. The CT scan did not show any sign of malignancy involving the urachal remnant.

The management of omphalitis/umbilical abscess that is associated with a urachal remnant consists of 2 sequential stages. The first stage focuses on treating the infection with antibiotics, as well as incision and drainage of the abscess. After the resolution of the infection, surgical excision of the urachal remnant is performed to prevent recurrence of the infection or the development of carcinoma $[3,7]$. This 2-staged approach has been 
shown to reduce postoperative complications and overall length of hospitalization when compared to immediate surgical management without treating the infection first [7]. Our patient took a course of penicillin and aminoglycoside for a period of 10 days showing major clinical improvement.

\section{Statement of Ethics}

This study was reviewed and the need for approval was waived by the Ethics Committee at Saint George Hospital University Medical Center. Written informed consent was obtained from the patient for publication of this case report and all accompanying images.

\section{Conflict of Interest Statement}

The authors have no conflicts of interest to declare.

\section{Funding Sources}

The authors declare that no funding was received for this study.

\section{Author Contributions}

Antonios Tawk, Ali Abdallah, and Paul Maouchi contributed to conception, design, analysis and interpretation of data; drafting and revising the article; and final approval. Joanna Salameh, Melissa Kyriakos, Ghadi Abboud, Mona Dagher, Peter Semaan, and Aida Metri contributed to design, analysis, and interpretation of data. Salem Khoury and Raja Ashou contributed to conception, editing, analysis, and final approval.

\section{Data Availability Statement}

All data generated or analyzed during this study are included in this article. Further inquiries can be directed to the corresponding author.

\section{References}

1 Cushing AH. Omphalitis: a review. Pediatr Infect Dis J. 1985;4(3):282-5.

2 Painter K, Gossman W. Omphalitis. Treasure Island, FL: StatPearls Publishing; 2020.

3 Kasiakou SK, Rafailidis PI, Rosmarakis ES, Falagas ME. Recurrent omphalitis in adults. Scand J Gastroenterol. 2004 Jan 1;39(10):1021-4.

4 Schoenwolf GC, Larsen WJ. Larsen's human embryology. 4th ed. Thoroughly rev. and updated. Philadelphia: Churchill Livingstone/Elsevier; 2009.

5 Wilson AL, Gandhi J, Seyam O, Rahmani B, Patel S, Joshi G, et al. Urachal anomalies: a review of pathological conditions, diagnosis, and management. Transl Res Anat. 2019;16:100041.

6 Gallagher P. Omphalitis. 2019. Available from: https://emedicine.medscape.com/article/975422-workup.

7 Elkbuli A, Kinslow K, Ehrhardt JD, Hai S, McKenney M, Boneva D. Surgical management for an infected urachal cyst in an adult: case report and literature review. Int J Surg Case Rep. 2019;57:130-3.

8 Ward TT, Saltzman E, Chiang S. Infected urachal remnants in the adult: case report and review. Clin Infect Dis. 1993 Jan 1;16(1):26-9.

\section{Karger'}


9 Tazi F, Ahsaini M, Khalouk A, Mellas S, Stuurman-Wieringa RE, Elfassi MJ, et al. Abscess of urachal remnants presenting with acute abdomen: a case series. J Med Case Rep. 2012 Jul 30;6(1):226.

10 Cacciarelli AA, Kass EJ, Yang SS. Urachal remnants: sonographic demonstration in children. Radiology. 1990 Feb 1;174(2):473-5.

11 Wan YL, Lee TY, Tsai CC, Chen SM, Chou FF. The role of sonography in the diagnosis and management of urachal abscesses. J Clin Ultrasound. 1991 May;19(4):203. 